Ernährung in der Pflegepraxis

\section{Aktualisierung des Expertenstandards}

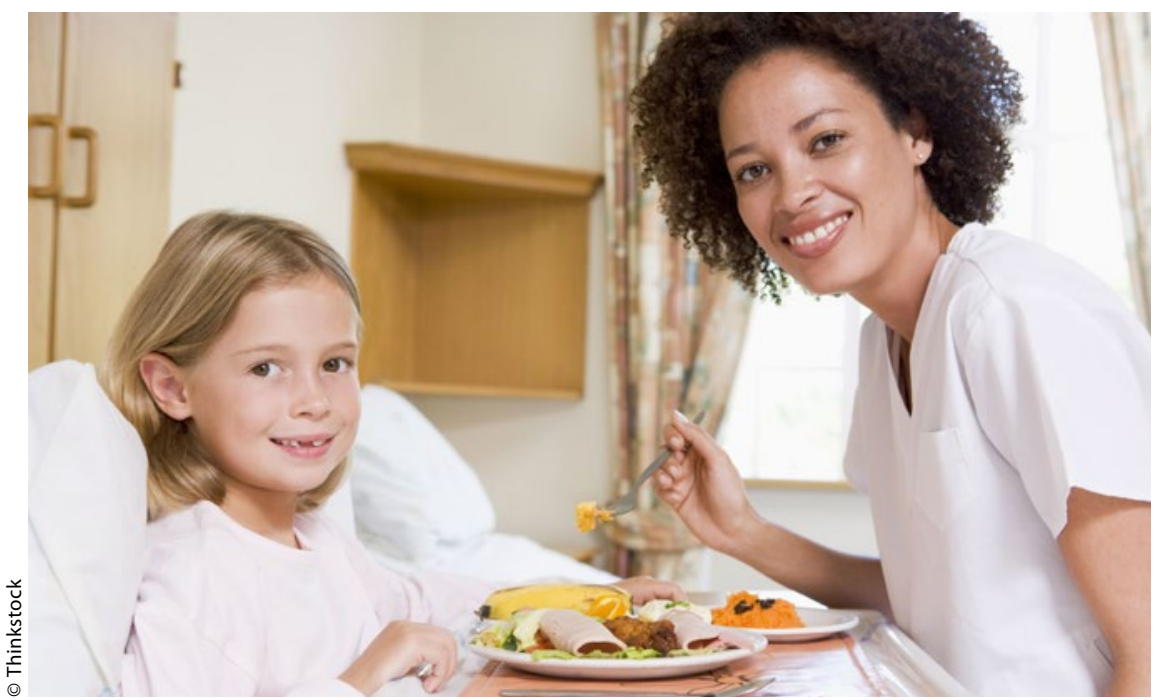

Im vergangenen Jahr hat das Deutsche Netzwerk für Qualitätsentwicklung in der Pflege (DNQP) mit der Aktualisierung des Expertenstandards „Ernährungsmanagement zur Sicherung und Förderung der oralen Ernährung in der Pflege" begonnen. Der jetzt vorliegende Entwurf basiert auf einer Analyse der aktuellen Literatur zum Thema und der eingehenden Diskussion des Wissensstandes durch eine zwölfköpfige Expertenarbeitsgruppe. Mit der Konsultationsfassung wird nun interessierten Personen aus Pflegepraxis und -wissenschaft sowie anderen Gesundheitsberufen die Möglichkeit gegeben, sich zur Aktuali- sierung zu äußern. Der Entwurf des aktualisierten Expertenstandards wird zusammen mit der Präambel, den Kommentierungen der Standardkriterien sowie der Literaturanalyse vom 1. Juni bis zum 29. Juli 2016 über die DNQP-Website einsehbar sein. Das DNQP bittet um schriftliche Rückmeldungen oder Stellungnahmen zur Konsultationsfassung per E-Mail oder Brief an die DNQP-Geschäftsstelle. Mit der Veröffentlichung des aktualisierten Expertenstandards ist im Herbst 2016 zu rechnen.

www.dnqp.de
Mehr Personal an der Charité Tarifkonflikt beigelegt

- Nach jahrelangen Auseinandersetzungen haben sich Charité - Universitätsmedizin Berlin und die Dienstleistungsgewerkschaft ver.di auf einen Tarifvertrag geeinigt. Bundesweit einmalig sind Vereinbarungen zur personellen Mindestbesetzung in der Intensivpflege, Kinderklinik und stationären Krankenpflege. In der Intensivpflege gilt eine hausinterne Qualitätsrichtlinie, die differenziert nach dem Schweregrad der Erkrankung, aber im Mittel aller Intensivstationen eine Mindestausstattung von einer Pflegekraft für zwei Patienten festlegt. Für die stationäre Pflege wurde eine Verbesserung der Pflegepersonalausstattung um $5 \%$ vereinbart, die bei besonderen Erschwernissen - wie Patienten mit multiresistenten Keimen - von zusätzlichem Personal ergänzt wird. Soweit keine anderen Kriterien gelten, werden für die Kinderklinik eine Besetzungsquote festgelegt und die Personalausstattung im Nachtdienst um 40 Vollkräfte verstärkt, um Unterstützung und Pausenregelungen zu gewährleisten. Zudem wurden Festlegungen für zahlreiche Funktionsbereiche, wie Operationssaal oder Endoskopie, getroffen.

Die Geschäftsführerin des Deutschen Berufsverbands für Pflegeberufe (DBfK) Nordost, Ulla Rose, spricht von einem „Meilenstein für die Pflegeberufe".

www.charite.de

Online-Umfrage zur Dienstplangestaltung

\title{
Brisante erste Ergebnisse veröffentlicht
}

— Im Rahmen seiner diesjährigen Aktion "Mein Recht auf Frei" hat der DBfK von Anfang März bis Mitte April 2016 eine Online-Umfrage zur Dienstplanung und Pausenregelung für Pflegende in Kliniken, Heimen und der ambulanten Pflege durchgeführt. 3.572 Antworten gingen ein und konnten in die Auswertung einbezogen werden, die momentan noch fortgesetzt wird. Aus Anlass des Internationalen Tages der Pflegenden wurden erste Ergebnisse veröffentlicht. Sie werfen ein grelles Schlaglicht auf die problematische Situation vor Ort und die Arbeitsbedingungen. Beispielsweise berichtet knapp die Hälfte der Umfrageteilnehmer, dass sie ein- bis zweimal im Monat kurzfristig Schichten übernehmen müssen, ein weiteres Drittel ist davon sogar dreibis fünfmal pro Monat und weitere $9 \%$ quasi wöchentlich betroffen.

Die Umfrage zeigt, dass freie Tage und
Urlaub häufig als kostenlose Rufbereitschaft betrachtet und die Mitarbeiter immer wieder zum Einspringen aufgefordert oder sogar genötigt werden. Gesetzlich vorgeschriebene Pausen entfallen, weil der Arbeitsdruck keine Pause zulässt. Die Folgen solcher Personalpolitik sind nicht zu übersehen: hohe Ausfallquoten, lange Krankheitsdauer, steigender Anteil psychischer Erkrankungen, Berufsflucht, schlechtes Berufsimage und sinkende Berufsverweildauer vor allem der jungen Generation.

www.dbfk.de 